\title{
Metabolic fuel selection: the importance of being flexible
}

\author{
Perry E. Bickel
}

Departments of Medicine and of Cell Biology \& Physiology, Division of Endocrinology, Metabolism and Lipid Research, Washington University School of Medicine, St. Louis, Missouri, USA.

\begin{abstract}
Studies in genetically engineered mice have shown the importance of crosstalk between organs in the regulation of energy metabolism. In this issue, a careful metabolic characterization of mice with genetic deficiency of the GLUT4 glucose transporter in adipocytes and muscle is reported (see the related article beginning on page 1666). These mice compensate for decreased peripheral glucose disposal by increasing hepatic glucose uptake and lipid synthesis as well as by increasing lipid utilization in peripheral tissues. These findings are relevant to humans with type 2 diabetes, in whom a key feature is diminished peripheral glucose disposal.
\end{abstract}

In type 2 diabetes, disposal of glucose into muscle and fat is diminished (1). After meals, increased blood glucose stimulates insulin secretion by pancreatic $\beta$ cells. Circulating insulin binds to insulin receptors on the surface of adipocytes and muscle cells and activates intracellular signaling pathways that promote the exocytosis of vesicles containing insulin-responsive GLUT4 (2). Increased numbers of glucose transporters on the cell surface facilitate transfer of glucose into cells, where it is trapped by phosphorylation and then further metabolized either for storage (as triglyceride or glycogen) or for energy (ATP) production. The mechanisms of insulin-regulated glucose transport have been studied extensively because glucose transport is rate-limiting for the metabolism of glucose in muscle and fat.

In addition to promoting glucose disposal and utilization in muscle and fat, insulin inhibits the production and release of glucose by the liver. However, resistance to insulin action in tissues (adipose, muscle, and liver) only leads to hyperglycemia and frank diabetes when $\beta$ cells fail to compensate sufficiently by increased insulin secretion and secretory capacity.

Nonstandard abbreviations used: AG4KO, adipose-specific GLUT4-KO; AMG4KO, adipose- and muscle-specific GLUT4-double KO; IRS2, insulin receptor substrate $2 ; \mathrm{MG} 4 \mathrm{KO}$, muscle-specific GLUT4-KO.

Conflict of interest: The author has declared that no conflict of interest exists.

Citation for this article: J. Clin. Invest. 114:1547-1549 (2004). doi:10.1172/JCI200423745.
Type 2 diabetes is a polygenic disease, so it is appealing to assume that multiple defects in multiple tissues act in additive or synergistic fashions to induce a resultant phenotype of hyperglycemia. Nevertheless, a reductionist model whereby defects in a single protein could lead to diabetes has been suggested by studies of insulin receptor substrate 2 (IRS2). Global KO of Irs2 in mice results in insulin resistance and failure of $\beta$ cell compensation, thereby leading to diabetes (3). Remarkably, $\mathrm{KO}$ of Irs 2 just in $\beta$ cells and parts of the brain is sufficient to reproduce the phenotype of the global $\mathrm{KO}$ animal $(4,5)$.

\section{Knockout of GLUT4 in fat and muscle of mice results in hyperglycemia, insulin resistance, and glucose intolerance}

In this issue of the JCI, Kotani, Kahn, and colleagues (6) describe another mouse model, an adipose- and muscle-specific GLUT4-double KO mouse (AMG4KO) that exhibits systemic effects on glucose homeostasis and fuel metabolism. The AMG4KO mouse represents an exaggerated model of the decreased glucose disposal into fat and muscle that occurs in type 2 diabetes. Kahn and colleagues had previously targeted GLUT4 individually in adipocytes (7), heart (8), and muscle (9) by Cre recombinase-mediated excision of exon 10 of the GLUT4 gene, which encodes the glucose-binding sites. Whereas cardiacspecific KO of GLUT4 resulted in cardiac hypertrophy but no systemic metabolic defects, adipose-specific and muscle-specific GLUT4-KO mice (hereafter desig- nated AG4KO and MG4KO, respectively) exhibited fasting hyperglycemia, insulin resistance, and glucose intolerance.

AG4KO and MG4KO mice demonstrate that diminished glucose uptake in fat or muscle results in secondary effects on the other insulin-responsive tissues, including the liver. GLUT4 deficiency in adipocytes results in insulin resistance in skeletal muscle and the liver, possibly due to an adipocyte-secreted factor (isolated muscles from AG4KO mice have normal insulinstimulated glucose uptake). GLUT4 deficiency in muscle leads to increased glucose uptake in liver as reflected by markedly increased glucose incorporation into liver glycogen. Thus one might expect that, in mice, GLUT4 deficiency in both adipocytes and muscle would lead to at least additive defects in whole-body glucose homeostasis. But, as Kotani et al. (6) report in this issue, this is not the case.

\section{Increased lipid utilization compensates for decreased glucose utilization in AMG4KO mice}

The AMG4KO mouse (generated by breeding aP2-Cre ${ }^{+/+}$GLUT4 lox ${ }^{+/-}$with MCK$\mathrm{Cre}^{+/+}$GLUT4 lox ${ }^{+/-}$mice) exhibits fasting hyperglycemia, insulin resistance, and glucose intolerance, but not to a degree significantly greater than either the AG4KO or MG4KO mouse (6). This observation led the authors to investigate what compensatory mechanisms come into play that limit the impact of the double hit on glucose homeostasis. Overall, AMG4KO mice display a shift in fuel utilization from glucose to lipid as revealed by a lower respiratory quotient and a blunted rise in respiratory quotient during feeding periods.

The authors detect significant changes in hepatic fuel metabolism that help explain this shift towards greater lipid utilization (6) (Figure 1). Specifically, hepatic uptake of glucose is increased, as suggested by increased levels of glucokinase protein in liver. What is the metabolic fate of this increased glucose load in the liver? Hepatic 

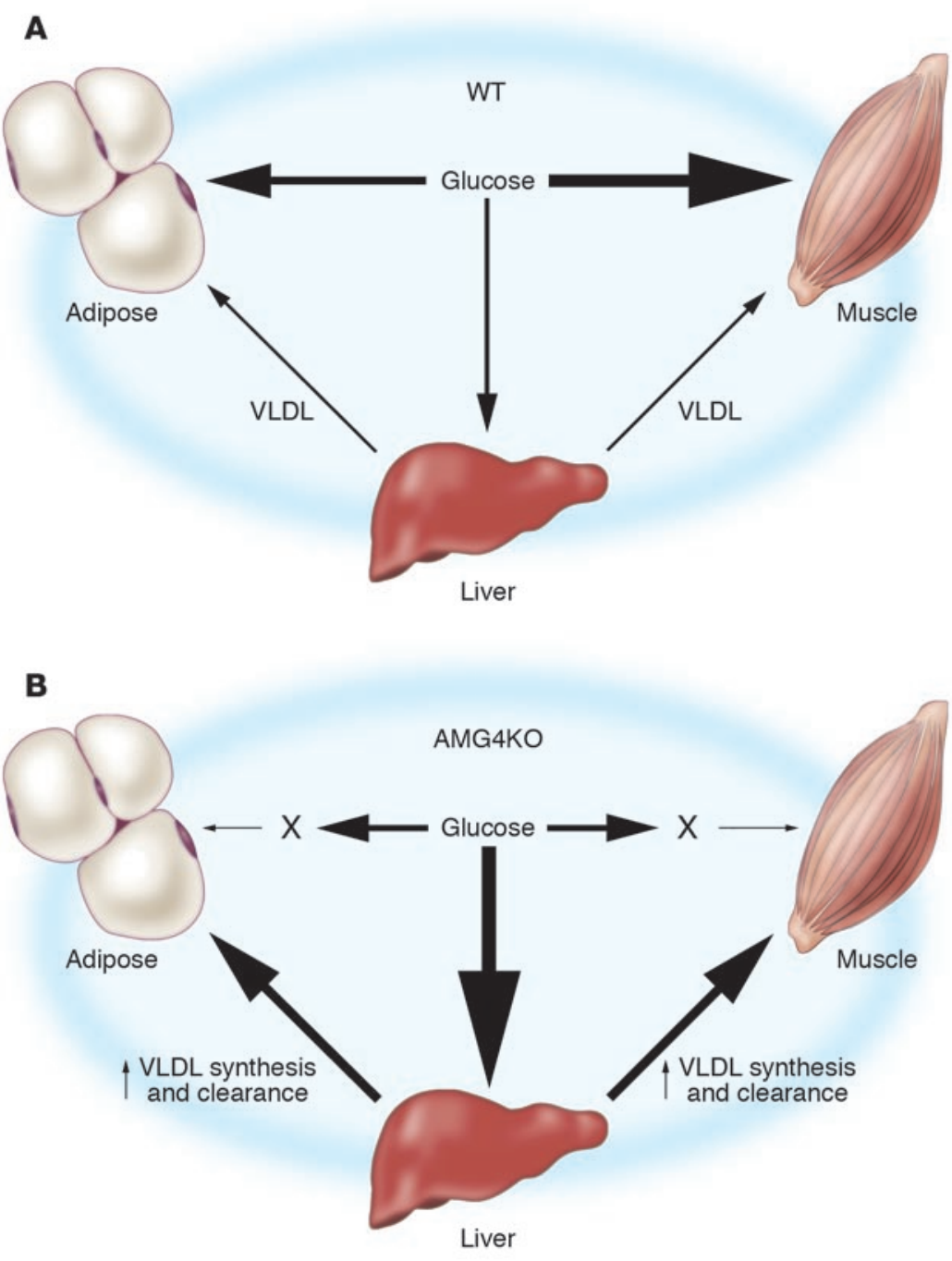

glycogen levels were not reported, although there is a trend towards lower whole-body glycogen synthesis compared with that in control littermates. However, glucose incorporation into de novo fatty acids is increased more than 3-fold compared with that in controls, and triglyceride synthesis in the liver is also increased. Increased conversion of glucose to fatty acids in the liver does not appear to result in increased triglyceride storage in liver, fat, or muscle, nor in increased steady-state serum levels of triglycerides or free fatty acids.

This leads us to ask the question: Where is the excess fat generated from the excess glucose going? This question remains unanswered experimentally by the current study (6), but the AMG4KO mice display increased clearance of triglycerides and free fatty acids following lipid loading by gavage. Thus, it is likely that there is increased fatty acid uptake and oxidation in muscle, which would provide a metabolic sink in which to dump excess fuel substrates. Whether fat oxidation in the adipose depot is upregulated to accommodate increased lipid flux remains to be determined. Future studies aimed at assessing levels of fat oxidation and expression of proteins involved in fatty acid transport and metabolism in AMG4KO mice should be instructive.

\section{Further questions raised by studies of AMG4KO mice}

What consequences does increased lipid flux through muscle have on the mouse? It will be important to evaluate motor activity and muscle performance, especially cardiac function, in AMG4KO mice as they age. The increased energy load on muscle does not result in storage as triglyceride but could be used for work. Alternatively, the energy load could be dissipated as heat generated through increased respiratory uncoupling or perhaps expended by cycles of triglyceride synthesis and hydrolysis or

\section{Figure 1}

Metabolic fuel selection in wild-type and AMG4KO mice. (A) In the fed state, wild-type mice dispose of glucose primarily in skeletal muscle and to a lesser extent in adipose tissue and liver. Glucose is stored by the liver as glycogen or used as a lipogenic substrate. Hepatic triglyceride is packaged into VLDL lipoproteins and secreted. Circulating triglycerides in VLDL are hydrolyzed by lipoprotein lipase in adipose and muscle to deliver fatty acid substrates for storage (in adipose tissue) or energy production (by muscle). (B) In AMG4KO mice, GLUT4 deficiency markedly attenuates glucose uptake in adipose and muscle, which leads to hyperglycemia. Hyperglycemia is limited by increased hepatic glucose uptake and metabolism into lipogenic substrates, via induction of glucokinase, SREBP-1c, and acetyl-CoA carboxylase. Triglyceride synthesis and, presumably, VLDL synthesis and secretion, are increased. However, circulating triglyceride and free fatty acid levels do not increase due to their increased clearance, presumably due to increased fat oxidation in peripheral tissues. some other futile energy cycle. Increased fatty acid oxidation has been associated with contractile dysfunction in isolated perfused hearts of $d b / d b$ mice (10), so there exists a precedent that a shift to increased lipid utilization in muscle may have deleterious effects in some metabolic contexts. Further exploration of such issues in the AMG4KO model may point to molecular mechanisms of metabolic adaptation that can be exploited for the development of new diabetes drugs.

In this study (6), the AMG4KO mice were only compared directly with AG4KO and MG4KO mice in terms of the results of glucose tolerance testing and fed insulin levels. While the AMG4KO mice were no more glucose intolerant than either the AG4KO or MG4KO mice, a subset of AMG4KO mice had significantly higher fed insulin levels, which suggests more severe insulin resistance. As noted by the authors, such a finding in this group of 
outbred mice provides a model system in which to identify genetic modifiers that contribute to insulin resistance.

Kotani et al. (6) have identified increased SREBP-1c, acetyl-CoA carboxylase, and glucokinase as mediators of the altered hepatic fuel metabolism observed in AMG4KO mice. It remains unknown how changes in glucose uptake in fat and muscle influence expression of these genes in the liver. Insulin and glucose can act coordinately to induce lipogenic enzymes via SREBP-1c and carbohydrate-responsive element-binding protein (11), so there may be no need to invoke any factors other than hyperinsulinemia and hyperglycemia. Indeed, in AMG4KO mice there were no significant changes in levels of plasma adipokines, such as adiponectin, though there was a trend toward increased resistin production. However, it is possible that adipokine levels in the portal vein may differ in the absence of changes in systemic levels.

The effects of decreased glucose disposal into fat and muscle on $\beta$ cell function in $A M G 4 K O$ mice remain to be explored. Hyperglycemia induced by partial pancreatectomy in rats leads to altered gene expression and decreased insulin secretory rate, and these changes are reversed by correction of hyperglycemia with phlorizin, which inhibits renal glucose reabsorption (12). Thus, the hyperglycemia observed in AMG4KO mice would likely inhibit insulin secretory capacity. Histologic and functional analysis of AMG4KO islets should reveal the extent of $\beta$ cell compensation or failure.

\section{The importance of metabolic flexibility in fuel selection}

The shift to lipid fuel utilization to compensate for diminished capacity for glucose uptake is attractive as a therapeutic strategy in type 2 diabetes. Unlike the AMG4KO mouse, humans with type 2 diabetes typically have elevated serum triglyceride and fatty acid levels and increased intramyocellular triglyceride. This may be due to what Kelley and Mandarino have termed "metabolic inflexibility," i.e., the inability to modulate the level of lipid oxidation to meet metabolic demand (13). Humans with type 2 diabetes also have reduced splanchnic glucose uptake, possibly due to defective glucokinase activity (14).

Can the conclusions drawn from studies of genetically engineered mice be applied to humans and human diseases such as diabetes? With respect to energy metabolism, differences between mice and humans may prevent a straightforward translation. For example, in mice, brown adipose tissue is essential for thermogenesis, but its role in adult humans is unclear. Also, mice have a significantly higher basal metabolic rate than humans and a markedly different circulating lipoprotein profile. And mice are short-lived. Problems related to increased lipid oxidation in muscle may not occur during the lifespan of the mouse but may pose significant adverse consequences in humans over time. Nevertheless, the work by Kotani, et al. (6) suggests targets for drug development. First, drugs that promote hepatic glucokinase activity (15) may help channel glucose into hepatocytes, where it can be used as a lipogenic substrate. Second, providing a metabolic sink for fatty acids in skeletal muscle would allow for increased flux of lipid into the muscle compartment to accommodate increased hepatic triglyceride synthesis and VLDL secretion. This potentially could be accomplished by stimulating fatty acid oxidation, e.g. pharmacologically with adiponectin proteins $(16,17)$ or by increasing uncoupling activity (18). Lest we focus entirely on speculative, and no doubt expensive, future therapies, there is one cheap, widely available, and underutilized therapy to stimulate muscle fatty acid oxidation - exercise (19).

\section{Acknowledgments}

The author thanks Nathan Wolins for helpful discussions during the preparation of this commentary.

Address correspondence to: Perry E. Bickel, Department of Medicine, Washington University School of Medicine, Campus Box 8127, 660 South Euclid Avenue, St. Louis, Missouri 63105, USA. Phone: (314) 747-3979; Fax: (314) 362-7641; E-mail: pbickel@im.wustl.edu.
1. Shepherd, P.R., and Kahn, B.B. 1999. Glucose transporters and insulin action-implications for insulin resistance and diabetes mellitus. N. Engl. J. Med. 341:248-257.

2. Bryant, N.J., Govers, R., and James, D.E. 2002. Regulated transport of the glucose transporter GLUT4. Nat. Rev. Mol. Cell Biol. 3:267-277.

3. Withers, D.J., et al. 1998. Disruption of IRS-2 causes type 2 diabetes in mice. Nature. 391:900-904.

4. Kubota, N., et al. 2004. Insulin receptor substrate 2 plays a crucial role in $\beta$ cells and the hypothalamus. J. Clin. Invest. 114:917-927. doi:10.1172/ JCI200421484.

5. Lin, X., et al. 2004. Dysregulation of insulin receptor substrate 2 in $\beta$ cells and brain causes obesity and diabetes. J. Clin. Invest. 114:908-916. doi:10.1172/JCI200422217.

6. Kotani, K., Peroni, O.D., Minokoshi, Y., Boss, O., and Kahn, B.B. 2004. GLUT4 glucose transporter deficiency increases hepatic lipid production and peripheral lipid utilization. J. Clin. Invest. 114:16661675. doi:10.1172/JCI200421341.

7. Abel, E.D., et al. 2001. Adipose-selective targeting of the GLUT4 gene impairs insulin action in muscle and liver. Nature. 409:729-733.

8. Abel, E.D., et al. 1999. Cardiac hypertrophy with preserved contractile function after selective deletion of GLUT4 from the heart. J. Clin. Invest. 104:1703-1714.

9. Zisman, A., et al. 2000. Targeted disruption of the glucose transporter 4 selectively in muscle causes insulin resistance and glucose intolerance. Nat. Med. 6:924-928.

10. Belke, D.D., Larsen, T.S., Gibbs, M., and Severson, D.L. 2000. Altered metabolism causes cardiac dysfunction in perfused hearts from diabetic $(d b / d b)$ mice. Am.J. Physiol. Endocrinol. Metab. 279:E1104-E1113.

11. Dentin, R., et al. 2004. Hepatic glucokinase is required for the synergistic action of ChREBP and SREBP-1c on glycolytic and lipogenic gene expression. J. Biol. Chem. 279:20314-20326.

12. Laybutt, D.R., et al. 2002. Genetic regulation of metabolic pathways in beta-cells disrupted by hyperglycemia. J. Biol. Chem. 277:10912-10921.

13. Kelley, D.E., and Mandarino, L.J. 2000. Fuel selection in human skeletal muscle in insulin resistance: a reexamination. Diabetes. 49:677-683.

14. Basu, A., et al. 2000. Effects of type 2 diabetes on the ability of insulin and glucose to regulate splanchnic and muscle glucose metabolism: evidence for a defect in hepatic glucokinase activity. Diabetes. 49:272-283.

15. Grimsby, J., et al. 2003. Allosteric activators of glucokinase: potential role in diabetes therapy. Science. 301:370-373.

16. Tomas, E., et al. 2002. Enhanced muscle fat oxidation and glucose transport by ACRP30 globular domain: acetyl-CoA carboxylase inhibition and AMP-activated protein kinase activation. Proc. Natl. Acad. Sci. U. S. A. 99:16309-16313.

17. Yamauchi, T., et al. 2002. Adiponectin stimulates glucose utilization and fatty-acid oxidation by activating AMP-activated protein kinase. Nat. Med. 8:1288-1295.

18. Li, B., et al. 2000. Skeletal muscle respiratory uncoupling prevents diet-induced obesity and insulin resistance in mice. Nat. Med. 6:1115-1120.

19. Goodpaster, B.H., Katsiaras, A., and Kelley, D.E. 2003. Enhanced fat oxidation through physical activity is associated with improvements in insulin sensitivity in obesity. Diabetes. 52:2191-2197. 